\title{
An impact of stochastic dynamic boundary conditions on the evolution of the Cahn-Hilliard system ${ }^{1}$
}

\author{
Abbreviated Title: Impact of stochastic dynamic boundary conditions \\ Desheng Yang \\ School of Mathematical Sciences and Computing Technology \\ Central South University, Changsha 410083, China \\ E-mail:dsyang@mail.csu.edu.cn \\ and \\ Jinqiao Duan (Corresponding author) \\ Department of Applied Mathematics, Illinois Institute of Technology Chicago, IL 60616, USA \\ E-mail:duan@iit.edu
}

\begin{abstract}
:
Nonlinear systems are often subject to random influences. Sometimes the noise enters the system through physical boundaries and this leads to stochastic dynamic boundary conditions. A dynamic, as opposed to static, boundary condition involves the time derivative as well as spatial derivatives for the system state variables on the boundary. Although stochastic static (Neumann or Dirichet type) boundary conditions have been applied for stochastic partial differential equations, not much is known about the dynamical impact of stochastic dynamic boundary conditions. The purpose of this paper is to study possible impacts of stochastic dynamic boundary conditions on the long term dynamics of the CahnHilliard equation arising in the materials science. We show that the dimension estimation of the random attractor increases as the coefficient for the dynamic term in the stochastic dynamic boundary condition decreases. However, the dimension of the random attractor is not affected by the corresponding stochastic static boundary condition.
\end{abstract}

\footnotetext{
${ }^{1}$ Date: July 25, 2006.

MSC 2000: Primary-60H15, 37L55; secondary-35R60,37H10.

Key words and phrases: Stochastic partial differential equations (SPDES), stochastic dynamic boundary condition, nonlinear system under uncertainty, microscopic mechanism on boundary, random dynamical system, random attractor.

Acknowledgment: We thank Alain Miranville and Dirk Blomker for helpful comments. This work was partly supported by the NSF Grants DMS-0209326 \& DMS-0542450.
} 


\section{Introduction}

The deterministic Cahn-Hilliard equation was introduced 4] as a mathematical model for the description of phase separation phenomena in materials such as binary alloys. The concentration $\phi$ of one of the two components of the binary alloy satisfies

$$
\partial_{t} \phi=\Delta(-\Delta \phi+f(\phi)) \text { in } \mathrm{G},
$$

where domain $G:=\Pi_{i=1}^{n}\left(0, L_{i}\right), L_{i}>0, n \in\{1,2,3\}$ and the boundary is denoted by $\Gamma$. The function $f$ is the derivative of a logarithmic potential which is usually approximated by a polynomial with strictly positive dominant coefficient. Theoretical results on asymptotic dynamical behavior of the system (1.1), under either Neumann or periodic boundary conditions, can be founded, for example, in the survey 24] or the book [30, and [9, 2, 18.

Recently physicists have considered phase separation phenomena in confined systems [16, 17, 20], where interactions with the walls have to be taken into account. This leads to dynamic boundary conditions, i.e., $\partial_{t} \phi$ appears in the boundary conditions. For example, one of the important phase separation phenomena when the binary alloy is suddenly cooled sufficiently is called spinodal decomposition. Once the effective interaction between the wall (i.e., the boundary $\Gamma$ ) and two components of the alloy are short-ranged in spinodal decomposition, the so-called dynamic boundary conditions have to be taken into account, together with the the Cahn-Hilliard equation. Mathematical results concerning the Cahn-Hilliard equation with (deterministic) dynamic boundary conditions have been obtained recently; see [23, 26, 32 . and references therein. We specially note that the so-called viscous Cahn-Hilliard equation with dynamic boundary conditions [23,

$$
\begin{aligned}
& \partial_{t} \phi=\Delta \mu, \mu=-\Delta \phi+\varepsilon \partial_{t} \phi+f(\phi), \text { in } G, \\
& \partial_{\nu} \mu=0, \text { on } \Gamma, \\
& \partial_{t} \phi=\Delta_{\|} \phi-\lambda \phi-\partial_{\nu} \phi-g(\phi), \text { on } \Gamma, \\
& \phi(0)=\phi_{0},
\end{aligned}
$$

where $\varepsilon \geq 0$ is a small parameter, $\Delta_{\|}$is the Laplace-Beltrami operator on the boundary $\Gamma, \mu$ is chemical potential, $\nu$ is the unit outer normal vector to $\Gamma$ and $\lambda$ is some given positive constant. Miranville and Zelik [23] have recently constructed a robust family of exponential attractors for this system when the nonlinear functions $f$ and $g$ are of arbitrary growths but satisfy some dissipativity assumptions.

Moreover, the environmental or surrounding fluctuations may also influence the system evolution and thus may be taken into account as well [2, 9, 15]. The present paper is concerned with the stochastic version of Eq. (1.2) with a stochastic dynamic 
boundary condition. It is given by

$$
\begin{aligned}
& d \phi=\Delta \mu d t+\sigma_{1} d W^{(1)}, \mu=-\Delta \phi+\varepsilon \partial_{t} \phi+f(\phi), \text { in } G, \\
& \partial_{\nu} \mu=0, \text { on } \Gamma, \\
& d \phi=\left(\Delta_{\|} \phi-\lambda \phi-\partial_{\nu} \phi-g(\phi)\right) d t+\sigma_{2} d W^{(2)}, \text { on } \Gamma, \\
& \phi(0)=\phi_{0},
\end{aligned}
$$

where $W^{(1)}$ and $W^{(2)}$ are independent Wiener processes which will be explained in detail later, and the constants $\sigma_{i}>0, i=1,2$, for the noise intensities. The random fluctuation terms consisting of $\left(W^{(1)}, W^{(2)}\right)$ act in the domain but also on the boundary $\Gamma$ and give a refined description of the underlying microscopic mechanism in the phase separation phenomena. To simplify the situation, we only consider the case with $g=0$, and the potential $f(u)$ is a polynomial of odd degree with a positive leading coefficient such as

$$
f(u)=\sum_{k=1}^{2 p-1} a_{k} u^{k}, a_{2 p-1}>0, p \geq 2 .
$$

There have only been a few works on spatially extended systems under stochastic dynamic boundary conditions [13, 5. It is intriguing to know the impact of such dynamic boundary conditions on the overall dynamics.

As in the deterministic case 23, we introduce another unknown function, namely, $\psi=\left.\phi\right|_{\Gamma}$, defined on the boundary $\Gamma$ and rewrite Eq.(1.3) as a coupled system of Itô parabolic stochastic partial differential equations (SPDEs) of the form:

$$
\begin{aligned}
& d \phi=\Delta \mu d t+\sigma_{1} d W^{(1)}, \mu=-\Delta \phi+\varepsilon \partial_{t} \phi+f(\phi), x \in G \\
& \partial_{\nu} \mu=0, \text { on } \Gamma \\
& \phi(0)=\phi_{0} \\
& d \psi=\left(\Delta_{\|} \psi-\lambda \psi-\partial_{\nu} \phi\right) d t+\sigma_{2} d W^{(2)}, x \in \Gamma \\
& \psi(0)=\psi_{0} \\
& \left.\phi\right|_{\Gamma}=\psi
\end{aligned}
$$

The aim of this paper is study the possible impact of the stochastic dynamic boundary condition on the overall evolution of the stochastic Cahn-Hilliard equation (1.5). To this end, we will, later on in Section 5 (see Remark 5.4), look at the dynamic boundary condition but with a positive intensity parameter $\varepsilon_{0}$ in front of the time derivative,

$$
\frac{1}{\varepsilon_{0}} d \psi=\left(\Delta_{\|} \psi-\lambda \psi-\partial_{\nu} \phi\right) d t+\sigma_{2} d W^{(2)}, x \in \Gamma .
$$


We will be able to see an impact of the stochastic dynamic boundary condition by looking at how the random attractor varies with the dynamic intensity parameter $\varepsilon_{0}$.

Based the theory on random dynamical systems [1, we first define a cocycle by the stochastic flow corresponding to the solution map for Eq.(1.5). Due to the additive noise, by introducing appropriate stochastic convolutions, we can solve the problem (1.5) pathwise, and thus construct a cocycle. As the cocycle is given, we then prove the system possesses a random attractor. Since the system considered is non-autonomous, we employ the pull-back technique to describe the asymptotic behavior. Finally we estimate Hausdorff dimension [11 of the random attractor and obtain its dependence on the dynamic intensity parameter $\varepsilon_{0}$.

This paper is arranged as follows. In Section 2, we present some preliminaries including some function spaces, inequalities, the spectrum results on some operators, the definitions of the noises and the basic framework of random dynamical systems. In Section 3, we construct a cocycle, defined via the stochastic flow generated by the stochastic Cahn-Hilliard equation (1.5). In Section 4, we prove the existence of the random attractor. Finally, in Section 5, we show that the dimension estimation of the random attractor increases as the coefficient for the dynamic term in the stochastic dynamic boundary condition decreases. However, the dimension of the random attractor is not affected by the corresponding stochastic static boundary condition.

\section{Preliminaries}

In this section we introduce some function spaces, inequalities, the known spectrum results on some operators [30, 23] and the definitions of the noises [10. For convenience, we also recall the basic framework of random dynamical systems theory [1] and random attractors.

\subsection{Function spaces and noises}

We denote by $H$ (respectively, $H(\Gamma)$ ) the usual Sobolev space $L^{2}(G)$ (respectively, $L^{2}(\Gamma)$ ) with the inner product $(\cdot, \cdot)$ (respectively, $\left.(\cdot, \cdot)_{\Gamma}\right)$ and the norm $|\cdot|$ (respectively, $\left.(|\cdot|)_{\Gamma}\right)$. Let $m(u)$ be the average over $G$

$$
m(u)=\frac{1}{|G|} \int_{G} u(x) d x
$$

and $H_{0}$ be a subspace of $H$ defined by

$$
H_{0}=\{u \in H: m(u)=0\} .
$$


We define the linear unbounded operator $A=-\Delta$ with domain $D(A)=\{u \in$ $H^{2}(G) \cap H_{0}: \partial_{\nu} u=0$ on $\left.\Gamma\right\}$. Then its spectrum consists of an infinite sequence of real eigenvalues $0=\lambda_{0}<\lambda_{1} \leq \lambda_{2} \leq \cdots \rightarrow \infty$. The corresponding eigenvectors $\left\{e_{i}^{(1)}\right\}$ form a complete orthonormal basis in $H$. Also notice that $\left\{e_{i}^{(1)}: i \in \mathbb{N}\right\}$ is a complete orthonormal basis in $H_{0}$ and the function $e_{0}^{(1)}$ is equal to a constant $|G|^{-1 / 2}$. By spectral theory, we may define the fractional powers $A^{s}$ of $A, s \in R$, by

$$
A^{s} u=\sum_{i=1}^{\infty} \lambda_{i}^{s} u_{i} e_{i}^{(1)},
$$

if $u=\sum_{i=0}^{\infty} u_{i} e_{i}^{(1)}$. The domain of $A^{s / 2}$ is

$$
V_{s}=D\left(A^{s / 2}\right)=\left\{u=\sum_{i=0}^{\infty} u_{i} e_{i}^{(1)}: \sum_{i=1}^{\infty} \lambda_{i}^{s} u_{i}^{2}<\infty\right\} .
$$

This domain space is endowed with the seminorm $|u|_{s}=\left|A^{s / 2} u\right|$, the semiscalar product $(u, v)_{s}=\left(A^{s / 2} u, A^{s / 2} v\right)$ and the norm:

$$
\|u\|_{s}=\left(|u|_{s}^{2}+m^{2}(u)\right)^{\frac{1}{2}} .
$$

Therefore, we have

$$
V_{0}=H, A^{0} u=\bar{u}=u-m(u),\|\bar{u}\|_{s}=|\bar{u}|_{s}=|u|_{s}
$$

and the following Poincare type inequality:

$$
|u|_{s} \leq \lambda_{1}^{\frac{s-t}{2}}|u|_{t}, s \leq t, u \in V_{t}
$$

together with the interpolation inequality:

$$
|u|_{\lambda s+(1-\lambda) t} \leq|u|_{s}^{\lambda}|u|_{t}^{1-\lambda}, s \leq t, \lambda \in[0,1], u \in V_{t} .
$$

We also define the operator $B$ by the Lalace-Beltrami operator, $B=-\Delta_{\|}$, with the domain $D(B)=H^{2}(\Gamma)$. Its spectrum consists of an infinite sequence of real eigenvalues $0<\mu_{1} \leq \mu_{2} \leq \cdots \rightarrow \infty$. The corresponding eigenvectors $\left\{e_{i}^{(2)}\right\}_{i \in \mathbb{N}}$ form a complete orthonormal basis in $H(\Gamma)$. Like the Sobolev space $V_{s}$ defined on $G$, we can define the space $V_{s, \Gamma}$ on $\Gamma$ with the seminorm $|\cdot|_{s, \Gamma}$ and the semiscalar product $(\cdot, \cdot)_{s, \Gamma}$, and also have the corresponding interpolation inequality and Poincare-type inequality.

For the additive stochastic term, we assume the following. 
Assumption 2.1. The stochastic process $W(t):=\left(W^{(1)}(t), W^{(2)}(t)\right)$, defined on a filtered probability space $\left(\Omega, \mathcal{F},\left\{\mathcal{F}_{t}\right\}_{t \in \mathbb{R}}, P\right)$, is a two-side in time Wiener process (Brownian motion) on $\left(H_{0}, H(\Gamma)\right)$, with covariance operator $Q=\left(Q_{1}, Q_{2}\right)$. It is given as the expansions

$$
W^{(j)}(t)=\sum_{i=1}^{\infty} \sqrt{\alpha_{i}^{(j)}} \beta_{i}^{(j)}(t) e_{i}^{(j)}, \quad \text { with } \quad Q_{j} e_{i}^{(j)}=\alpha_{i}^{(j)} e_{i}^{(j)}, j=1,2
$$

where $\left\{\left(e_{i}^{(1)}, e_{i}^{(2)}\right)\right\}_{i \in \mathbb{N}}$ are the orthonormal basis of $\left(H_{0}, H(\Gamma)\right)$ from the eigenvectors of the operator $A$ and B. Moreover, $\left\{\left(\beta_{i}^{(1)}, \beta_{i}^{(2)}\right)\right\}_{i \in \mathbb{N}}$ are independent (two-sided in time) standard scalar Wiener processes (Brownian motions) on the probability space $(\Omega, \mathcal{F}, P)$. It is also assumed that the covariance operator $Q=\left(Q_{1}, Q_{2}\right)$ is a HilbertSchmidt operator, and is of trace class, i.e. $\operatorname{tr} Q_{j}=\sum_{i=1}^{\infty} \alpha_{i}^{(j)}<\infty, j=1,2$.

The special case $Q=I$, where $I$ is an identity operator, or equivalently, $\alpha_{i}^{(j)}=$ $1, j=1,2$ for all $i \in \mathbb{N}$, corresponds to the case of a cylindrical white noise (white in both time and space). Therefore, the cylindrical Wiener process does not satisfy the above assumption and our result in this paper does not apply to this case. In the deterministic case the Cahn-Hilliard equation preserves the spatial average of $\phi$ in time. According to the definition of $W(t)$, we have $\int_{G} W^{(1)}(t, x) d x=0$. It guarantees that the white noise does not destroy this property, i.e., the spatial average of $\phi$ is still a conversed quantity:

$$
m(\phi(t))=m(\phi(0)) .
$$

Let us consider the operator $A_{\varepsilon}$ defined by

$$
A_{\varepsilon} u=\left(\varepsilon+A^{-1}\right)^{-1} A u,
$$

for all $u \in D\left(A_{\varepsilon}\right)=D(A)$. Then the spectrum of $A_{\varepsilon}$ consists of eigenvalues $r_{k}$ :

$$
r_{k}=\left(\varepsilon+\lambda_{k}^{-1}\right)^{-1} \lambda_{k}=\frac{\lambda_{k}^{2}}{1+\varepsilon \lambda_{k}},
$$

and the corresponding eigenfunction $e_{k}^{(1)}$.

Under Assumption 2.1 the linear equation

$$
\begin{aligned}
& d z+\Delta^{2} z d t=\varepsilon \Delta d z+\sigma_{1} d W^{(1)}, x \in G, \\
& \left.\partial_{\nu} z\right|_{\Gamma}=0,
\end{aligned}
$$

which can be rewritten as the abstract form:

$$
\left(\varepsilon+A^{-1}\right) d z+A z d t=\sigma_{1} A^{-1} d W^{(1)}(t), \text { in } H_{0},
$$


has a unique stationary solution given by the Itô integral and Fourier series expansion

$$
\begin{aligned}
z(t) & =\sigma_{1} \int_{-\infty}^{t} e^{-A_{\varepsilon}(t-s)}(I+\varepsilon A)^{-1} d W^{(1)}(s) \\
& =\sigma_{1} \sum_{k=1}^{+\infty} \frac{\sqrt{\alpha_{k}^{(1)}}}{\varepsilon \lambda_{k}+1} \int_{-\infty}^{t} e^{-r_{k}(t-s)} d \beta_{k}^{(1)} e_{k}^{(1)},
\end{aligned}
$$

which is also called as a stochastic convolution. As is well known, $z(t)$ is a continuous Gaussian process in the space $H_{0}$. Moreover, we can prove the process $z(t, x)$ satisfies the following regularity (similar to [9] and [10]).

Throughout the paper, the letters $C$ and $C_{i}$ denote some generic constants, which may change from one line to another.

Lemma 2.2. Under Assumption 2.1, the process $\nabla z(t, x)$ has a version which is $\nu$-Hölder continuous with respect to $(t, x) \in \mathbb{R} \times G$, for any $\nu \in\left[0, \frac{1}{4}\right), \varepsilon \geq 0$. Furthermore, if $\varepsilon>0$, we have $A z(t, x) \in C^{\nu}(\mathbb{R} \times G)$, for any $\nu \in\left[0, \frac{1}{2}\right)$.

Proof. For the sake of simplicity, we assume $G=(0, \pi)$. Therefore, the eigenfunctions $\left\{e_{i}^{(1)}: i \in \mathbb{N}\right\}$ satisfy

$$
\left|e_{i}^{(1)}\right| \leq 1, \quad\left|\nabla e_{i}^{(1)}\right| \leq \sqrt{\lambda_{i}}
$$

From the representation (2.4), we can write $\nabla z$ as the following expansion

$$
\nabla z(t, x)=\sigma_{1} \sum_{k=1}^{+\infty} \frac{\sqrt{\alpha_{k}^{(1)}}}{1+\varepsilon \lambda_{k}} \int_{-\infty}^{t} e^{-r_{k}(t-s)} d \beta_{k}^{(1)} \nabla e_{k}^{(1)}
$$

Therefore, using the inequality $\left|\nabla e_{k}^{(1)}(x)-\nabla e_{k}^{(1)}(y)\right| \leq \lambda_{k}|x-y|$, we have the estimate for any $x, y \in G, \varepsilon \geq 0$,

$$
\begin{aligned}
E|\nabla z(t, x)-\nabla z(t, y)|^{2} & \leq \sigma_{1}|x-y|^{2} \sum_{k=1}^{+\infty} \frac{\alpha_{k}^{(1)} \lambda_{k}^{2}}{\left(1+\varepsilon \lambda_{k}\right)^{2}} \int_{-\infty}^{t} e^{-2 r_{k}(t-s)} d s \\
& =\frac{\sigma_{1}}{2}|x-y|^{2} \sum_{k=1}^{+\infty} \frac{\alpha_{k}^{(1)}}{1+\varepsilon \lambda_{k}} \leq \frac{\sigma_{1}}{2} \operatorname{tr} Q_{1}|x-y|^{2}
\end{aligned}
$$

Furthermore, using the inequality $\left|A e_{k}^{(1)}(x)-A e_{k}^{(1)}(y)\right| \leq \lambda_{k}^{\frac{3}{2}}|x-y|$, for $\varepsilon>0$, we obtain

$$
\begin{aligned}
E|A z(t, x)-A z(t, y)|^{2} & \leq \sigma_{1}|x-y|^{2} \sum_{k=1}^{+\infty} \frac{\alpha_{k}^{(1)} \lambda_{k}^{3}}{\left(1+\varepsilon \lambda_{k}\right)^{2}} \int_{-\infty}^{t} e^{-2 r_{k}(t-s)} d s \\
& =\frac{\sigma_{1}}{2}|x-y|^{2} \sum_{k=1}^{+\infty} \frac{\alpha_{k}^{(1)} \lambda_{k}}{1+\varepsilon \lambda_{k}} \leq \frac{\sigma_{1}}{2 \varepsilon} \operatorname{tr} Q_{1}|x-y|^{2} .
\end{aligned}
$$


Moreover, fixing $t>s, x \in G$, we have

$$
\begin{aligned}
& E|\nabla z(t, x)-\nabla z(s, x)|^{2} \\
\leq & \sigma_{1} \sum_{k=1}^{+\infty} \frac{\alpha_{k}^{(1)} \lambda_{k}}{\left(1+\varepsilon \lambda_{k}\right)^{2}}\left\{\int_{s}^{t} e^{2 r_{k}(\tau-t)} d \tau+\int_{-\infty}^{s}\left|e^{(\tau-t) r_{k}}-e^{(\tau-s) r_{k}}\right|^{2} d \tau\right\} \\
= & \sigma_{1} \sum_{k=1}^{+\infty} \frac{\alpha_{k}^{(1)} \lambda_{k}}{\left(1+\varepsilon \lambda_{k}\right)^{2} r_{k}}\left\{1-e^{r_{k}(s-t)}\right\} .
\end{aligned}
$$

Writing $C_{\beta}=\sup _{x \geq 0, y \geq 0} \frac{e^{-x}-e^{-y}}{|x-y|^{2 \beta}}$, we get for $\beta \in\left[0, \frac{1}{4}\right], \varepsilon \geq 0$,

$$
\begin{aligned}
E|\nabla z(t, x)-\nabla z(s, x)|^{2} & \leq C \sum_{k=1}^{+\infty} \frac{\alpha_{k}^{(1)} \lambda_{k}}{\left(1+\varepsilon \lambda_{k}\right)^{2}} r_{k}^{2 \beta-1}|t-s|^{2 \beta} \\
& =C \sum_{k=1}^{+\infty} \frac{\alpha_{k}^{(1)} \lambda_{k}^{4 \beta-1}}{\left(1+\varepsilon \lambda_{k}\right)^{2 \beta+1}}|t-s|^{2 \beta} \leq C|t-s|^{2 \beta} .
\end{aligned}
$$

Similarly, for $\varepsilon>0, t>s, \beta \in\left[0, \frac{1}{2}\right]$, we have

$$
E|A z(t, x)-A z(s, x)|^{2} \leq C \sum_{k=1}^{+\infty} \frac{\alpha_{k}^{(1)} \lambda_{k}^{4 \beta}}{\left(1+\varepsilon \lambda_{k}\right)^{2 \beta+1}}|t-s|^{2 \beta} \leq C \varepsilon^{-2 \beta-1}|s-t|^{2 \beta} .
$$

Consequently, for $\beta \in\left[0, \frac{1}{4}\right], \varepsilon \geq 0$, by (2.5) and (2.7), there exists a positive constant $C$ such that

$$
E|\nabla z(t, x)-\nabla z(s, y)|^{2} \leq C\left(|x-y|^{2}+|t-s|^{2}\right)^{\beta},
$$

for $x, y \in G, t, s \in \mathbb{R}$. Since the random variable $\nabla z(t, x)-\nabla z(s, y)$ is Gaussian, then for all $m \in N$, we obtain

$$
E\left(|\nabla z(t, x)-\nabla z(s, y)|^{2 m}\right) \leq C\left(|x-y|^{2}+|t-s|^{2}\right)^{m \beta},
$$

which gives that $\nabla z(t, x) \in C^{\nu}(\mathbb{R} \times G)$, for any $\nu \in\left[0, \frac{1}{4}\right), \varepsilon \geq 0$ from Kolmogorov Theorem ([25]). Similarly, by (2.6) and (2.8), for $\varepsilon>0$, we have $A z(t, x) \in C^{\nu}(\mathbb{R} \times$ $G), \nu \in\left[0, \frac{1}{2}\right)$.

Remark 2.3. Note that the estimates for eigenfunctions, $\left|e_{i}^{(1)}\right| \leq 1$ and $\left|\nabla e_{i}^{(1)}\right| \leq$ $\sqrt{\lambda_{i}}$, are true for rectangular and triangular domains, but not true for disks. It is unknown which geometrical conditions (on the domain) imply these estimates. See detailed discussions in [12, [3].

\subsection{Random dynamical systems}

Let $(\Omega, \mathcal{F}, P)$ be a probability space and $\left\{\theta_{t}: \Omega \rightarrow \Omega, t \in \mathbb{R}\right\}$ a family of measurepreserving transformations such that $\theta_{0}=I$, and $\theta_{t+s}=\theta_{t} \circ \theta_{s}$, for all $t, s \in R$. Here $\left\{\theta_{t}\right\}$ is called a metric or driving dynamical system on $(\Omega, \mathcal{F}, P)$. We always assume that $\theta$ is ergodic under probability measure $P$. 
Definition 2.4. Let $(X, d)$ be a Polish space (i.e., complete separable metric space). A measurable map

$$
\varphi: \mathbb{R} \times \Omega \times X \rightarrow X, \quad(t, \omega, x) \mapsto \varphi(t, \omega) x,
$$

is called a random dynamical system $(\boldsymbol{R D} \boldsymbol{S})$ if $\varphi$ satisfies the cocycle property: $\varphi(0, \omega)=I, \varphi(t+s, \omega)=\varphi\left(t, \theta_{s} \omega\right) \varphi(s, \omega)$, for all $t, s \in \mathbb{R}$ and P-a.s. $\omega \in \Omega$.

A RDS is said to be continuous if $\varphi(t, \omega): X \rightarrow X$ is continuous, P-a.s. for $\omega$ and for every $t \in \mathbb{R}$. Notice that the $\operatorname{RDS} \varphi$ defined above is two-sided in time and invertible, $\varphi(t, \omega)^{-1}=\varphi\left(-t, \theta_{t} \omega\right)$ P-a.s. As random attractors are characterized by random sets, we have to deal with some new concepts such as absorbing sets, attraction and invariance. Specially the pull-back approach, starting from $-\infty$ and observing time 0 , has been extensively explored in the theory of random dynamical systems. Before defining them, we recall a compact random set as defined below.

Definition 2.5. A set-valued map $K: \Omega \rightarrow 2^{X}$, the set of all subsets of $X$, is called a random compact set if $K(\omega)$ is a compact P-almost surely and if $\omega \rightarrow d(x, K(\omega))$ is measurable for each $x \in X$, where $d(x, M):=\inf _{y \in M} d(x, y)$.

The pull-back technique is used to define the following concepts.

Definition 2.6. Let $A(\omega)$ and $B(\omega)$ be two random sets. We say

(1). $A(\omega)$ attracts $B(\omega)$ if $P$-a.s.,

$$
\lim _{t \rightarrow \infty} \operatorname{dist}\left(\varphi\left(t, \theta_{-t} \omega\right) B\left(\theta_{-t} \omega\right), A(\omega)\right)=0,
$$

where $\operatorname{dist}(\cdot, \cdot)$ denotes the Hausdorff semidistance in $X$, defined as $\operatorname{dist}(A, B)=$ $\sup _{x \in A} \inf _{y \in B} d(x, y)$, for $\forall A, B \subset X$.

(2). $A(\omega)$ absorbs $B(\omega)$ if there exists $t_{B}(\omega)$ such that for all $t \geq t_{B}(\omega)$,

$$
\varphi\left(t, \theta_{-t} \omega\right) B\left(\theta_{-t} \omega\right) \subset A(\omega) .
$$

holds P-a.s.

It is clear that a random absorbing set is attracting. We call a invariant compact attracting set as a random attractor. Formally [6, 28,

Definition 2.7. A random set $\mathcal{A}(\omega)$ is called a random attractor for the $R D S \varphi$ if P-a.s.

(1). $\mathcal{A}(\omega)$ is a random compact set.

(2). $\mathcal{A}(\omega)$ is strictly invariant, i.e., $\varphi(t, \omega) \mathcal{A}(\omega)=\mathcal{A}\left(\theta_{t} \omega\right)$, for $\forall t \geq 0$. 
(3). $\mathcal{A}(\omega)$ attracts all bounded deterministic sets in $X$.

The existence result of random attractors is stated as follows (e.g., [6, 8, 28, 29]).

Theorem 2.8. If there exists a random compact set absorbing every bounded nonrandom set $B \subset X$, then the $R D S \varphi$ possesses a random attractor $\mathcal{A}(\omega)$

$$
\mathcal{A}(\omega)=\overline{\bigcup_{B \subset X} \Lambda_{B}(\omega)}
$$

where $\Lambda_{B}(\omega):=\bigcap_{s \geq 0 t \geq s} \overline{\bigcup_{s} \varphi\left(t, \theta_{-t} \omega\right) B}$ is the omega-limit set of $B$.

\section{Remark 2.9.}

(1). In fact, a random attractor $\mathcal{A}(\omega)$ in Definition 2.6 is a global random set attractor which is uniquely determined by a attracting compact set ([7]).

(2). $\varphi\left(t, \theta_{-t} \omega\right) x$ can be interpreted as the position at $t=0$ of the trajectory which was at $x$ at time $-t$, that is, while time $t$ is moving, the trajectory $\varphi\left(t, \theta_{-t} \omega\right) x$ is always at the position at time zero. Therefore, the random attractor in Definition 2.7 is also called as the "pullback attractor".

One of the results in the theory of global attractors for deterministic systems is that the Hausdorff dimension of the attractor is often finite. Although the random attractor is not uniformly bounded, it is expected that the techniques on the Hausdorff dimension of a global attractor of a deterministic system can be generalized to the stochastic case under some assumptions [11, 29]. In fact, based on Laypunov exponents, Debussche [11] showed that the Hausdorff dimension of a random attractor is finite if the corresponding cocycle $\varphi(t, \omega)$ satisfies some properties, especially uniformly differentiability. The following conclusion is due to Debussche [11.

Theorem 2.10. Let $\mathcal{A}(\omega)$ be a compact measurable set which is invariant under a random map $S(\omega), \omega \in \Omega$, for some ergodic metric dynamical system $\left(\Omega, \mathcal{F}, P,\left(\theta_{t}\right)_{t \in \mathbb{R}}\right)$. Assume that

1. $S(\omega)$ is almost surely uniformly differentiable on $\mathcal{A}(\omega)$, that is, for every $u, u+$ $h \in \mathcal{A}(\omega)$, there exists $D(S(\omega ; u))$ in $\mathcal{L}(H)$, the space of bounded linear operator from a Hilbert space $H$ to itself, such that

$$
|S(\omega)(u+h)-S(\omega) u-D S(\omega ; u) h| \leq \bar{k}(\omega)|h|^{1+\mu},
$$

where $\mu>0, \bar{k}(\omega)$ is a random variable satisfying $\bar{k}(\omega) \geq 1, E(\log \bar{k})<\infty$. 
2. $\omega_{d}(D S(\omega, u)) \leq \bar{\omega}_{d}(\omega)$ for $u \in \mathcal{A}(\omega)$ and some random variable $\bar{\omega}_{d}(\omega)$ satisfying $E\left(\log \left(\bar{\omega}_{d}\right)\right)<0$, where

$$
\omega_{d}(L)=\alpha_{1}(L) \cdots \alpha_{d}(L), \alpha_{i}(L)=\inf _{\substack{F \subset H \\ \operatorname{dim} F \leq i-1}} \sup _{\substack{\varphi \in F^{\perp} \\|\varphi|_{H}=1}}|L \varphi|_{H} \quad \text { for } \quad L \in \mathcal{L}(H) .
$$

3. $\alpha_{1}(D S(\omega, u)) \leq \bar{\alpha}_{1}(\omega)$, for $u \in \mathcal{A}(\omega)$ and a random variable $\bar{\alpha}_{1}(\omega) \geq 1$ with $E\left(\log \bar{\alpha}_{1}\right)<\infty$.

Then the Hausdorff dimension $d_{H}(\mathcal{A}(\omega))$ of $\mathcal{A}(\omega)$ is less than $d$ almost surely.

\section{Stochastic flow}

The stochastic Cahn-Hilliard equation with stochastic dynamical boundary conditions is non-autonomous, and thus it is impossible to define a semigroup on the phase space. In fact, a solution of the stochastic equation gives a stochastic flow instead of a semigroup. In this section, we introduce stochastic convolutions to solve the problem (1.5) pathwise and obtain the corresponding stochastic flow. In fact, the stochastic flow satisfies the so-called cocycle property and thus leads to a RDS modelling our system.

Let $\Omega$ be the set of continuous functions with value $(0,0) \in \mathbb{R}^{2}$ at 0

$$
\Omega=\left\{\omega \in C\left(\mathbb{R}, \mathbb{R}^{2}\right): \omega(0)=(0,0)\right\}
$$

Let $\mathcal{F}$ be the Borel sigma-algebra induced by the compact-open topology of $\Omega$, and $P$ a Wiener measure on $(\Omega, \mathcal{F})$. We write

$$
W(t)=\left(W^{(1)}(t), W^{(2)}(t)\right)=\left(\omega^{(1)}(t), \omega^{(2)}(t)\right)=\omega(t), t \in \mathbb{R}, \omega \in \Omega,
$$

and define

$$
\theta_{t} \omega(s)=\omega(t+s)-\omega(t), \quad t \in \mathbb{R} .
$$

It is easy to get $\theta_{t} \circ \theta_{s}=\theta_{t+s}$ in terms of (2.1). Thus $\left(\Omega, \mathcal{F}, P,\left(\theta_{t}\right)_{t \in \mathbb{R}}\right)$ is an ergodic metric dynamical system which models the white noise.

Following [23], we introduce the weak energy space $\mathbb{L}_{\varepsilon}$ with the norm

$$
|(\phi, \psi)|_{\mathbb{L}_{\varepsilon}}^{2}=\varepsilon|\phi|^{2}+\|\phi\|_{-1}^{2}+|\psi|_{\Gamma}^{2},
$$

and the phase space $\mathbb{V}$ with the norm

$$
|(\phi, \psi)|_{\mathbb{V}}^{2}=\|\phi\|_{1}^{2}+\|\psi\|_{1, \Gamma}^{2} .
$$


For convenience, using the operator $A^{-1}: H_{0} \rightarrow H_{0}$, we rewrite the system (1.5) as follows:

$$
\begin{aligned}
& \left(\varepsilon+A^{-1}\right) d \phi=\left(\Delta \phi-<\partial_{\nu} \phi>_{\Gamma}-\bar{f}(\phi)\right) d t+\sigma_{1} A^{-1} d W^{(1)}, x \in G, \\
& \phi(0)=\phi_{0}, \\
& d \psi=\left(\Delta_{\|} \psi-\lambda \psi-\partial_{\nu} \phi\right) d t+\sigma_{2} d W^{(2)}, x \in \Gamma, \\
& \psi(0)=\psi_{0}, \\
& \left.\phi\right|_{\Gamma}=\psi,
\end{aligned}
$$

where $\left\langle u>_{\Gamma}=\frac{1}{|G|} \int_{\Gamma} u(x) d x\right.$ and $\bar{f}=f-m(f)$.

Let $z^{(1)}$ and $z^{(2)}$ be stationary solutions of (2.3) and the linear equation

$$
d z=\left(\Delta_{\|} z-\lambda z\right) d t+\sigma_{2} d W^{(2)}, x \in \Gamma,
$$

respectively. Similar to Lemma 2.2 we can obtain $\nabla_{\|} z^{(2)}(t, x) \in C^{\nu}(\mathbb{R} \times \Gamma), \nu \in$ $\left[0, \frac{1}{4}\right)$.

Set $(u, v)=\left(\phi-z^{(1)}, \psi-z^{(2)}\right)$. Then $(u, v)$ satisfies

$$
\begin{aligned}
& \left(\varepsilon+A^{-1}\right) \partial_{t} u=\Delta u-<\partial_{\nu} u>_{\Gamma}-\bar{f}\left(u+z^{(1)}\right), x \in G \\
& u(0)=\phi_{0}-z^{(1)}(0) \\
& \partial_{t} v=\Delta_{\|} v-\lambda v-\partial_{\nu} u, x \in \Gamma \\
& v(0)=\psi_{0}-z^{(2)}(0) \\
& \left.u\right|_{\Gamma}=v+z^{(2)}-\left.z^{(1)}\right|_{\Gamma}
\end{aligned}
$$

where we have used the fact $\left.\partial_{\nu} z^{(1)}\right|_{\Gamma}=0$.

According to [23], the system (3.2) possesses a unique solution $(u, v) \in C\left([s, t], \mathbb{L}_{\varepsilon}\right)$, for every initial value $(u(s), v(s)) \in \mathbb{L}_{\varepsilon}, s \in \mathbb{R}, t \geq s, \omega \in \Omega$, and so, there exists a continuous operator $S_{\varepsilon}(t, s ; \omega)$ on the weak energy space $\mathbb{L}_{\varepsilon}$ :

$$
S_{\varepsilon}(t, s ; \omega): \mathbb{L}_{\varepsilon} \rightarrow \mathbb{L}_{\varepsilon}, S_{\varepsilon}(t, s ; \omega)(\phi(s), \psi(s))=(\phi(t), \psi(t)) .
$$

The corresponding stochastic flow can be defined by

$$
\varphi(t, \omega)(\phi(0), \psi(0))=S_{\varepsilon}(t, 0 ; \omega)(\phi(0), \psi(0)) .
$$

Notice that P-a.s.,

$$
S_{\varepsilon}(t, s ; \omega)=S_{\varepsilon}(t, r ; \omega) S_{\varepsilon}(r, s ; \omega), S_{\varepsilon}(t, s ; \omega)=S_{\varepsilon}\left(t-s, 0 ; \theta_{s} \omega\right) .
$$

It implies that $\varphi$ satisfies the cocycle property. In the end, $\varphi$ gives a continuous random dynamical system on $\mathbb{L}_{\varepsilon}$ over $\left(\Omega, \mathcal{F}, P,\left(\theta_{t}\right)_{t \in \mathbb{R}}\right)$ associated with the stochastic Cahn-Hilliard equation (3.1) under stochastic dynamic boundary condition. 


\section{The random attractor}

In this section, we prove the stochastic Cahn-Hilliard equations (3.1) possesses a random attractor. We first recall that the stochastic system (3.1) possesses the conservation law (2.2) and, consequently, we cannot expect to construct a random attractor in the whole phase space $\mathbb{L}_{\varepsilon}$. Therefore, we work in the affine space

$$
\mathbb{L}_{\varepsilon}^{\beta}=\left\{u \in \mathbb{L}_{\varepsilon}: m(u)=\beta, 0<\varepsilon \leq 1\right\},
$$

with the norm $\mathbb{L}_{\varepsilon}$, and prove the restriction of the cocycle $(\phi, \psi)$ on the $\mathbb{L}_{\varepsilon}^{\beta}$ possesses a compact absorbing set. By Theorem 2.8, we conclude that the stochastic CahnHilliard equations (3.1) with stochastic dynamic boundary conditions has a random attractor.

We begin by proving the existence of a absorbing set in $\mathbb{L}_{\varepsilon}^{\beta}$.

Lemma 4.1. Given any ball of $\mathbb{L}_{\varepsilon}, B(0, \rho)$ centered at 0 of radius $\rho$, for any $-1 \leq$ $t \leq 1, \omega \in \Omega$, there exist random variables $R_{t}(\omega)$ and $t(\rho ; \omega)<-2$ such that for any $s \leq t(\rho ; \omega),\left(\phi_{s}, \psi_{s}\right) \in B(0, \rho)$,

$$
\left|S_{\varepsilon}(t, s ; \omega)\left(\phi_{s}, \psi_{s}\right)\right| \leq R_{t}(\omega),
$$

holds P-a.s.

Proof. Let $(u(t), v(t))=\left(u\left(t, \omega ; s, u_{s}\right), v\left(t, \omega ; s, v_{s}\right)\right)$ be the solution to Eqs. (3.2) with the initial value $\left(u_{s}, v_{s}\right)=\left(\phi_{s}-z^{(1)}(s), \psi_{s}-z^{(2)}(s)\right)$. Multiplying the first equation of (3.2) by $\bar{u}$ and integrating over $G$, we obtain

$$
\frac{1}{2} \partial_{t}\left(\varepsilon|\bar{u}|^{2}+|u|_{-1}^{2}\right)=(\Delta u, \bar{u})-\left(\bar{f}\left(u+z^{(1)}\right), \bar{u}\right),
$$

where we have used $m(\bar{u})=0$. We denote $\bar{v}=v-m(u)$ and estimate the first term of the right hand side of Eq.(4.2) as follows:

$$
\begin{aligned}
(\Delta u, \bar{u}) & =\left(-\partial_{t} v+\Delta_{\|} v-\lambda v, \bar{v}\right)_{\Gamma}+\left(\partial_{\nu} u, z^{(2)}-z^{(1)}\right)_{\Gamma}-|u|_{1}^{2} \\
& =-|u|_{1}^{2}-\frac{1}{2} \partial_{t}|\bar{v}|_{\Gamma}^{2}-\left|\nabla_{\|} v\right|_{\Gamma}^{2}-\lambda|v|_{\Gamma}^{2}+\lambda(v, m(u))_{\Gamma}+\left(\partial_{\nu} u, z^{(2)}-z^{(1)}\right)_{\Gamma} .
\end{aligned}
$$

Notice that

$$
\lambda(v, m(v))_{\Gamma} \leq \frac{\lambda}{2}|v|_{\Gamma}^{2}+C \beta^{2}
$$

and

$$
\begin{aligned}
\left(\partial_{\nu} u, z^{(2)}-z^{(1)}\right)_{\Gamma} & \leq\left\|\partial_{\nu} u\right\|_{-\frac{1}{2}, \Gamma}\left\|z^{(2)}-z^{(1)}\right\|_{\frac{1}{2}, \Gamma} \\
& \leq \frac{1}{4}\|u\|_{1}^{2}+\left(\left\|z^{(2)}\right\|_{\frac{1}{2}, \Gamma}+\left\|z^{(1)}\right\|_{\frac{3}{2}}\right)^{2}
\end{aligned}
$$


Therefore, we have

$$
(\Delta u, \bar{u}) \leq-\frac{1}{2} \partial_{t}|\bar{v}|_{\Gamma}^{2}-\left|\nabla_{\|} v\right|_{\Gamma}^{2}-\frac{\lambda}{2}|v|_{\Gamma}^{2}-\frac{3}{4}|u|_{1}^{2}+C \beta^{2}+\left(\left\|z^{(2)}\right\|_{\frac{1}{2}, \Gamma}+\left\|z^{(1)}\right\|_{\frac{3}{2}}\right)^{2} .
$$

Since $a_{2 p-1}>0$, we see that

$$
f(x) x \geq \frac{1}{2} a_{2 p-1} x^{2 p}-C,|f(x)| \leq 2 a_{2 p-1}|x|^{2 p-1}+C,
$$

for some positive constant $C$. Therefore, we have the estimate on the second term of the right hand side of Eq.(4.2):

$$
\begin{aligned}
-\left(\bar{f}\left(u+z^{(1)}\right), \bar{u}\right) & =-\left(f\left(u+z^{(1)}\right), u+z^{(1)}-m(u)-z^{(1)}\right) \\
& \left.\leq-\frac{a_{2 p-1}}{2} \mid u+z^{(1)}\right)\left.\right|_{L^{2 p}} ^{2 p}+\left(f\left(u+z^{(1)}\right), m(u)+z^{(1)}\right)+C,
\end{aligned}
$$

and

$$
\begin{aligned}
\left(f\left(u+z^{(1)}\right), m(u)+z^{(1)}\right) & \leq 2 a_{2 p-1} \int_{G}\left(\left|u+z^{(1)}\right|^{2 p-1}+C\right)\left|z^{(1)}+m(u)\right| d x \\
& \leq \frac{1}{8} a_{2 p-1}\left|u+z^{(1)}\right|_{L^{2 p}}^{2 p}+C\left(\left|z^{(1)}\right|_{L^{2 p}}^{2 p}+\beta^{2 p}+1\right) .
\end{aligned}
$$

In the end, we obtain

$$
-\left(\bar{f}\left(u+z^{(1)}\right), \bar{u}\right) \leq-\frac{3 a_{2 p-1}}{8}\left|u+z^{(1)}\right|_{L^{2 p}}^{2 p}+C\left(\left|z^{(1)}\right|_{L^{2 p}}^{2 p}+\beta^{2 p}+1\right) .
$$

Summing (4.3) and (4.4) into (4.2), for $\varepsilon \in(0,1]$, we obtain

$$
\begin{aligned}
& \partial_{t}\left(\varepsilon|\bar{u}|^{2}+|u|_{-1}^{2}+|\bar{v}|_{\Gamma}^{2}\right)+\frac{\lambda_{1} \varepsilon}{4}|\bar{u}|^{2}+\frac{\lambda_{1}^{2}}{4}|u|_{-1}^{2}+\lambda|v|_{\Gamma}^{2} \\
& +|u|_{1}^{2}+2|v|_{1, \Gamma}^{2}+\frac{3 a_{2 p-1}}{4}\left|u+z^{(1)}\right|_{L^{2 p}}^{2 p} \\
\leq & C\left(\left\|z^{(1)}\right\|_{\frac{3}{2}}^{2 p}+\left\|z^{(2)}\right\|_{\frac{1}{2}, \Gamma}^{2}+\beta^{2 p}+1\right)
\end{aligned}
$$

where we have used inequalities $|u|_{-1} \leq \lambda_{1}^{-1}|u|_{1},|\bar{u}|^{2} \leq \lambda_{1}^{-1}|u|_{1}^{2}$ and the Sobolev embedding theorem $V_{\frac{3}{2}} \hookrightarrow L^{2 p}(n=1,2,3)$.

Denoting $\kappa=\min \left\{\frac{\lambda_{1}}{4}, \frac{\lambda_{1}^{2}}{4}, \lambda\right\}>0$ and applying the Grownwall lemma to (4.5), we obtain

$$
\begin{aligned}
& |(\bar{u}(t), \bar{v}(t))|_{\mathbb{L}_{\varepsilon}^{\beta}}^{2}+\int_{s}^{t} e^{-\kappa(t-\tau)}\left(|(u(\tau), v(\tau))|_{\mathbb{V}}^{2}+\frac{3}{4} a_{2 p-1}\left|u+z^{(1)}\right|_{L^{2 p}}^{2 p}\right) d \tau \\
\leq & |(\bar{u}(s), \bar{v}(s))|_{\mathbb{L}_{\varepsilon}^{\beta}}^{2} e^{-\kappa(t-s)}+C \int_{s}^{t} e^{-\kappa(t-\tau)}\left(\left\|z^{(1)}\right\|_{\frac{3}{2}}^{2 p}+\left\|z^{(2)}\right\|_{\frac{1}{2}, \Gamma}^{2}+\beta^{2 p}+1\right) d \tau .
\end{aligned}
$$

Thus, for any ball $B(0, \rho) \subset \mathbb{L}_{\varepsilon}^{\beta}$ and $(\phi(s), \psi(s)) \in B(0, \rho)$, there exists a random variable $t(\rho ; \omega)<-2$ such that for all $s<t(\rho ; \omega), t \in[-1,1]$,

$$
\begin{aligned}
& |(u(t), v(t))|_{\mathbb{L}_{\varepsilon}^{\beta}}^{2}+\int_{s}^{t}|(u(\tau), v(\tau))|_{\mathbb{V}}^{2} d \tau+\int_{s}^{t}\left|u(\tau)+z^{(1)}(\tau)\right|_{L^{2 p}}^{2 p} d \tau \\
\leq & C\left(1+\beta^{2 p}+\left|\left(z^{(1)}(t), z^{(2)}(t)\right)\right|_{\mathbb{L}_{\varepsilon}}^{2}+\int_{-\infty}^{t} e^{-\kappa(t-\tau)}\left(\left\|z^{(1)}\right\|_{\frac{3}{2}}^{2 p}+\left\|z^{(2)}\right\|_{\frac{1}{2}, \Gamma}^{2}\right) d \tau\right) .
\end{aligned}
$$


Denote by $r_{t}^{2}(\omega)$ the right hand side of the above inequality, which is finite P-a.s. by Lemma 2.2. Writing $R_{t}^{2}(\omega)=2\left(r_{t}^{2}(\omega)+\left|\left(z^{(1)}(t), z^{(2)}(t)\right)\right|_{\mathbb{L}_{\varepsilon}}^{2}\right)$, we complete the proof of the lemma.

Lemma 4.1 shows that for any deterministic bounded set $B \subset B(0, \rho)$ in $\mathbb{L}_{\varepsilon}^{\beta}$, there exists a random time $t(\rho ; \omega)<-2$ such that for any $s<t(\rho ; \omega)$,

$$
S_{\varepsilon}(-1, s ; \omega) B \subset B\left(0, R_{-1}(\omega)\right) .
$$

Noticing that

$$
\varphi\left(-s, \theta_{s} \omega\right)=S_{\varepsilon}\left(-s, 0 ; \theta_{s} \omega\right)=S_{\varepsilon}(0, s ; \omega)=S_{\varepsilon}(0,-1 ; \omega) S_{\varepsilon}(-1, s ; \omega),
$$

we find that $\mathcal{B}(\omega):=S_{\varepsilon}(0,-1 ; \omega) B\left(0, R_{-1}(\omega)\right)$ is a random absorbing set in $\mathbb{L}_{\varepsilon}^{\beta}$. Moreover, we can prove $\mathcal{B}(\omega)$ is compact. In order to do it, we first give some estimates.

Multiplying the first equation of (3.2) by $A u$ and integrating over $G$, we obtain

$$
\frac{1}{2} \partial_{t}\left(\varepsilon|u|_{1}^{2}+|\bar{u}|^{2}\right)+|u|_{2}^{2}=-\left(\bar{f}\left(u+z^{(1)}\right), A u\right)
$$

It is possible to find a positive constant $C$ such that

$$
f^{\prime}(u)>\frac{2 p-1}{2} a_{2 p-1} u^{2 p-2}-C,
$$

and

$$
\left|f^{\prime}(u)\right| \leq 2(2 p-1) a_{2 p-1} u^{2 p-2}+C,
$$

and thus, it gives

$$
\begin{aligned}
& -\left(f\left(u+z^{(1)}\right), A u\right) \\
= & -\int_{G} \nabla f\left(u+z^{(1)}\right) \nabla u d x \\
\leq & -\frac{1}{4}(2 p-1) a_{2 p-1} \int_{G}|u|^{2 p-2}|\nabla u|^{2} d x+C|u|_{1}^{2}+C_{1}\left(\left|u+z^{(1)}\right|_{L^{2 p}}^{2 p}+\left|\nabla z^{(1)}\right|_{L^{2 p}}^{2 p} .\right.
\end{aligned}
$$

Finally, (4.8) yields

$$
\begin{aligned}
& \partial_{t}\left(\varepsilon|u|_{1}^{2}+|\bar{u}|^{2}\right)+2|u|_{2}^{2}+\frac{(2 p-1) a_{2 p-1}}{2} \int_{G}|u|^{2 p-2}|\nabla u|^{2} d x \\
\leq & C|u|_{1}^{2}+C_{1}\left(\left|u+z^{(1)}\right|_{L^{2 p}}^{2 p}+\left|\nabla z^{(1)}\right|_{L^{2 p}}^{2 p}\right) .
\end{aligned}
$$

For any $t \in[-1,0], s<-1$, we have

$$
\begin{aligned}
& \varepsilon|u(t)|_{1}^{2}+|\bar{u}(t)|^{2} \\
\leq & \varepsilon|u(s)|_{1}^{2}+|\bar{u}(s)|^{2}+C \int_{s}^{t}|u(\tau)|_{1}^{2} d \tau+C_{1} \int_{s}^{t}\left(\left|u(\tau)+z^{(1)}(\tau)\right|_{L^{2 p}}^{2 p}+\left|\nabla z^{(1)}(\tau)\right|_{L^{2 p}}^{2 p}\right) d \tau .
\end{aligned}
$$


Integrating over $[-2,-1]$ on $s$ leads to

$$
\varepsilon|u(t)|_{1}^{2}+|\bar{u}(t)|^{2} \leq C \int_{-2}^{-1}|u(t)|_{1}^{2} d t+C_{1} \int_{-2}^{-1}\left(\left|u+z^{(1)}\right|_{L^{2 p}}^{2 p}+\left|\nabla z^{(1)}\right|_{L^{2 p}}^{2 p}\right) d t,
$$

which yields

$$
\begin{aligned}
\int_{-1}^{0}|u(t)|_{2}^{2} d t & \leq \varepsilon|u(-1)|_{1}^{2}+|\bar{u}(-1)|^{2}+C \int_{-1}^{0}|u(t)|_{1}^{2} d t+C_{1} \int_{-1}^{0}\left(\left|u+z^{(1)}\right|_{L^{2 p}}^{2 p}+\left|\nabla z^{(1)}\right|_{L^{2 p}}^{2 p}\right) d t \\
& \leq C \int_{-2}^{0}|u(t)|_{1}^{2} d t+C_{1} \int_{-2}^{0}\left(\left|u+z^{(1)}\right|_{L^{2 p}}^{2 p}+\left|\nabla z^{(1)}\right|_{L^{2 p}}^{2 p}\right) d t .
\end{aligned}
$$

Therefore, by (4.7), for $s<t(\rho ; \omega)$, we obtain

$$
\sup _{t \in[-1,0]}\left|u\left(t, \omega ; s, u_{s}\right)\right|<\infty, \int_{-1}^{0}\left|u\left(t, \omega ; s, u_{s}\right)\right|_{2}^{2} d t<\infty .
$$

The Sobolev embedding theorem $V_{2} \hookrightarrow L^{r}$ for $r \geq 2, n<4$, and the interpolation approach imply that for $q \in[2, \infty)$ and $\beta \in[2, \infty)$,

$$
\int_{-1}^{0}|u(t)|_{L^{q}}^{\beta} d t<\infty, \text { and thus, } \int_{-1}^{0}|\phi(t)|_{L^{q}}^{\beta} d t<\infty .
$$

Now we state that $\mathcal{B}(\omega)$ satisfies the following compact property.

Lemma 4.2. The random set $\mathcal{B}(\omega)$ described above is a compact absorbing set, that is, it is compact and absorbs any non-random bounded set: for every bounded deterministic set $B \subset B(0, \rho)$, we have

$$
\varphi\left(-s, \theta_{s} \omega\right) B \subset \mathcal{B}(\omega),
$$

holds P-a.s., for any $s \leq t(\rho ; \omega), t(\rho ; \omega)$ defined in Lemma 4.1.

Proof. We only need to prove that the random absorbing set $\mathcal{B}(\omega)$ is compact. Let $\left\{\left(\phi_{0}^{n}, \psi_{0}^{n}\right): n \in \mathbb{N}\right\}$ be a sequence in $\mathcal{B}(\omega)$ and $\left(u^{n}, v^{n}\right)$ a solution to Eqs. (3.2) with the initial value $\left(u^{n}(0), v^{n}(0)\right)=\left(\phi_{0}^{n}-z^{(1)}(0), \psi_{0}^{n}-z^{(2)}(0)\right)$. We easily get, from (4.5), that for any $t \in[-1,0]$

$$
\begin{aligned}
& \left|\left(u^{n}(t), v^{n}(t)\right)\right|_{\mathbb{L}_{\varepsilon}^{\beta}}^{2}+\int_{-1}^{t}|(u(\tau), v(\tau))|_{\mathbb{V}}^{2} d \tau \\
\leq & |(u(-1), v(-1))|_{\mathbb{L}_{\varepsilon}^{\beta}}^{2}+C\left(1+\beta^{2 p}+\int_{-1}^{t}\left(\left\|z^{(1)}\right\|_{\frac{3}{2}}^{2 p}+\left\|z^{(2)}\right\|_{\frac{1}{2}, \Gamma}^{2}\right) d \tau\right) .
\end{aligned}
$$

Since $|(u(-1), v(-1))|_{\mathbb{L}_{\varepsilon}^{\beta}}^{2} \leq r_{-1}^{2}(\omega)$, we deduce that $\left\{\left(u^{n}, v^{n}\right): n \in \mathbb{N}\right\}$ is bounded in $L^{\infty}\left(-1,0 ; \mathbb{L}_{\varepsilon}^{\beta}\right) \cap L^{2}(-1,0 ; \mathbb{V})$, and so, it is compact in $L^{2}\left(-1,0 ; \mathbb{L}_{\varepsilon}^{\beta}\right)$. Hence, there exists a subsequence $\left\{\left(u^{n_{k}}, v^{n_{k}}\right): n \in \mathbb{N}\right\}$ convergent to a function $(u, v)$ in 
$L^{2}\left(-1,0 ; \mathbb{L}_{\varepsilon}^{\beta}\right)$. Moveover $(u, v)$ is a solution of Eqs. (3.2). Let $\left(\phi_{0}, \psi_{0}\right)=(u(0)+$ $\left.z^{(1)}(0), v(0)+z^{(2)}(0)\right)$, it is easy to yield

$$
\phi_{0}-\phi_{0}^{n_{k}}=u(0)-u^{n_{k}}(0), \psi_{0}-\psi_{0}^{n_{k}}=v(0)-v^{n_{k}}(0) .
$$

We now prove the subsequence $\left\{\left(\phi_{0}^{n_{k}}, \psi_{0}^{n_{k}}\right)\right\}$ is convergent to $\left(\phi_{0}, \psi_{0}\right)$ in $\mathbb{L}_{\varepsilon}^{\beta}$. Writing $(X(t), Y(t))=\left(u^{n_{k}}(t)-u(t), v^{n_{k}}(t)-v(t)\right)$, then $(X(t), Y(t))$ satisfies the following equations

$$
\begin{aligned}
& \left(\varepsilon+A^{-1}\right) \partial_{t} X=\Delta X-<\partial_{\nu} X>_{\Gamma}-\left(\bar{f}\left(u^{n_{k}}+z^{(1)}\right)-\bar{f}\left(u+z^{(1)}\right)\right), x \in G, \\
& X(0)=\phi_{0}^{n_{k}}-\phi_{0} \\
& \partial_{t} Y=\Delta_{\|} Y-\lambda Y-\partial_{\nu} X, x \in \Gamma \\
& Y(0)=\psi_{0}^{n_{k}}-\psi_{0} \\
& \left.X\right|_{\Gamma}=Y .
\end{aligned}
$$

Multiplying the first equation of (4.12) by $\bar{X}$ and integrating over $G$, we obtain

$$
\frac{1}{2} \partial_{t}\left(\varepsilon|\bar{X}|_{1}^{2}+|X|_{-1}^{2}\right)=(\Delta X, \bar{X})-\left(\bar{f}\left(u^{n_{k}}+z^{(1)}\right)-\bar{f}\left(u+z^{(1)}\right), \bar{X}\right) .
$$

The two terms of the right hand side of (4.13) satisfy the following estimates, respectively,

$$
\begin{aligned}
& (\Delta X, \bar{X}) \leq-\frac{1}{2} \partial_{t}|\bar{Y}|_{\Gamma}^{2}-\left|\nabla_{\|} Y\right|_{\Gamma}^{2}-\frac{\lambda}{2}|Y|_{\Gamma}^{2}-|X|_{1}^{2}+C m^{2}(X), \\
& -\left(\bar{f}\left(u^{n_{k}}+z^{(1)}\right)-\bar{f}\left(u+z^{(1)}\right), \bar{X}\right) \\
& \leq C|X|^{2}+C_{1}|m(X)||X|\left(1+\left|u^{n_{k}}+z^{(1)}\right|_{4 p-4}^{4 p-4}+\left|u+z^{(1)}\right|_{4 p-4}^{4 p-4}\right),
\end{aligned}
$$

where we have used the fact $f^{\prime}(u) \geq-C$, and, consequently, from (4.12) and the estimate $|m(X)| \leq C|X|$, we deduce

$$
\begin{aligned}
\partial_{t}|(X(t), Y(t))|_{\mathbb{L}_{\varepsilon}^{\beta}}^{2} & \leq C\left(1+\left|u^{n_{k}}+z^{(1)}\right|_{L^{4 p-4}}^{4 p-4}+\left|u+z^{(1)}\right|_{L^{4 p-4}}^{4 p-4}\right)|X|^{2} \\
& \leq C\left(1+\left|u^{n_{k}}+z^{(1)}\right|_{L^{4 p-4}}^{4 p-4}+\left|u+z^{(1)}\right|_{L^{4 p-4}}^{4 p-4}\right)|X|_{\mathbb{L}_{\varepsilon}^{\beta}}^{2} \\
& :=C(t)|X|_{\mathbb{L}_{\varepsilon}^{\beta}}^{2},
\end{aligned}
$$

which leads to

$$
|(X(0), Y(0))|_{\mathbb{L}_{\varepsilon}^{\beta}}^{2} \leq|(X(t), Y(t))|_{\mathbb{L}_{\varepsilon}^{\beta}}^{2} e^{\int_{t}^{0} C(s) d s} .
$$

Integrating the above inequality over $[-1,0]$, we obtain

$$
|(X(0), Y(0))|_{\mathbb{L}_{\varepsilon}^{\beta}}^{2} \leq e^{\int_{-1}^{0} C(s) d s}|(X(t), Y(t))|_{L^{2}\left(-1,0 ; \mathbb{L}_{\varepsilon}^{\beta}\right)}^{2},
$$

which implies that the sequence $\left\{\left(\phi_{0}^{n_{k}}, \psi_{0}^{n_{k}}\right)\right\}$ converges to $\left(\phi_{0}, \psi_{0}\right)$, and thus, $\mathcal{B}(\omega)$ is compact.

Finally, using Theorem 2.8, we conclude 
Theorem 4.3. The random dynamical system associated with the stochastic CahnHilliard equation (1.5) with stochastic dynamic boundary conditions possesses a random attractor $\mathcal{A}_{\varepsilon}(\omega)$.

A compact absorbing set with the $\omega$-wise attraction property guarantees the existence of the random attractor, but the union in $\omega$ of $\mathcal{A}_{\varepsilon}(\omega)$ is not compact in general. Since $P$ is invariant under $\theta_{t}$, the forward attraction property from 0 to $\infty$ can be obtained w.r.t. convergence in probability, that is,

$$
\lim _{t \rightarrow \infty} P\left(\operatorname{dist}\left(\varphi(t, \omega) B, \mathcal{A}_{\varepsilon}\left(\theta_{t} \omega\right)\right)<\eta\right)=1
$$

holds for any $\eta>0$ and all deterministic bounded set $B \subset \mathbb{L}_{\varepsilon}^{\beta}$. This means the trajectories starting from $B$ at time 0 is always attracted by the moving compact set $\mathcal{A}_{\varepsilon}\left(\theta_{t} \omega\right)$.

\section{Impact of stochastic dynamic boundary conditions}

To investigate the impact of the stochastic dynamic boundary conditions on overall evolution, we estimate the Hausdorff dimension of the random attractor associated with the stochastic Cahn-Hilliard equations (1.5) or (3.1). We hope to find a upper bound of the Hausdorff dimension of the random attractor $\mathcal{A}_{\varepsilon}(\omega)$ by using Theorem 2.10 .

Define a random map $S$ in $\mathcal{L}_{\varepsilon}^{\beta}$ by

$$
S(\omega)=S_{\varepsilon}(1,0 ; \omega)=\varphi(1, \omega),
$$

and an ergodic transformation $\theta=\theta_{1}$. Then the random attractor $\mathcal{A}_{\varepsilon}(\omega), \omega \in \Omega$ is a compact measurable set invariant by $S$. In order to apply Theorem 2.10 we need to check that the three assumptions of this theorem hold. To establish the uniform differentiability of the random map $S$, we first give some estimates.

Let $\left(\phi_{i}(t), \psi_{i}(t)\right)(i=1,2)$ be two solutions of the problem (3.1) with the initial values $\left(\phi_{i}(0), \psi_{i}(0)\right)=\left(\phi_{i}^{0}, \psi_{i}^{0}\right) \in \mathbb{L}_{\varepsilon}^{\beta}$ and denote $(g(t), h(t))=\left(\phi_{1}(t)-\phi_{2}(t), \psi_{1}(t)-\right.$ $\left.\psi_{2}(t)\right)$. Then $(g(t), h(t))$ solves

$$
\begin{aligned}
& \left(\varepsilon+A^{-1}\right) \partial_{t} g(t)=\Delta g-<\partial_{\nu} g>_{\Gamma}-\left(\bar{f}\left(\phi_{1}\right)-\bar{f}\left(\phi_{2}\right)\right), x \in G, \\
& g(0)=\phi_{1}^{0}-\phi_{2}^{0}, \\
& \partial_{t} h(t)=\Delta_{\|} h-\lambda h-\partial_{\nu} g, x \in \Gamma, \\
& h(0)=\psi_{1}^{0}-\psi_{2}^{0}, \\
& \left.g\right|_{\Gamma}=h .
\end{aligned}
$$


Taking the scalar product in $H$ of the first equation of (5.1) with $g$, by $m(g)=0$ and $f^{\prime}(u)>-C$, we get

$$
\partial_{t}|(g, h)|_{\mathbb{L}_{\varepsilon}^{\beta}}^{2}+2|(g, h)|_{\mathbb{V}}^{2} \leq C|g|^{2} \leq \frac{1}{2}|g|_{1}^{2}+C|g|_{-1}^{2},
$$

which leads to

$$
|(g(t), h(t))|_{\mathbb{L}_{\varepsilon}^{\beta}}^{2} \leq e^{C t}|(g(0), h(0))|_{\mathbb{L}_{\varepsilon}^{\beta}}^{2} \text {, and } \int_{0}^{1}|(g(t), h(t))|_{\mathbb{V}}^{2} d t \leq C|(g(0), h(0))|_{\mathbb{L}_{\varepsilon}^{\beta}}^{2}
$$

The uniform differentiability of the random map $S$ can be stated as follows.

Lemma 5.1. $S(\omega)$ is almost surely uniformly differentiable on $\mathcal{A}_{\varepsilon}(\omega)$ : for $\left(\phi_{0}, \psi_{0}\right),\left(\phi_{0}+\right.$ $\left.h, \psi_{0}+l\right) \in \mathcal{A}_{\varepsilon}(\omega)$, there exists $D S\left(\omega ; \phi_{0}, \psi_{0}\right) \in \mathcal{L}\left(\mathbb{L}_{\varepsilon}^{0}\right)$ such that

$$
\left|S(\omega)\left(\phi_{0}+h, \psi_{0}+l\right)-S(\omega)\left(\phi_{0}, \psi_{0}\right)-D S\left(\omega ; \phi_{0}, \psi_{0}\right)(h, l)\right|_{\mathbb{L}_{\varepsilon}^{\beta}} \leq \bar{k}(\omega)|(h, l)|_{\mathbb{L}_{\varepsilon}^{\beta}}^{1+\mu},
$$

holds P-a.s., where $\mu>0, \bar{k}(\omega) \geq 1, E(\log \bar{k}(\omega))<\infty, D S\left(\omega ; \phi_{0}, \psi_{0}\right)(h, l)=(\Phi(1), \Psi(1))$, and $(\Phi(t), \Psi(t))$ is a solution to the first variation system of the system (3.1)

$$
\begin{aligned}
& \left(\varepsilon+A^{-1}\right) \partial_{t} \Phi=\Delta \Phi-<\partial_{\nu} \Phi>_{\Gamma}-\overline{f^{\prime}(\phi) \Phi}, x \in G, \\
& \Phi(0)=h \\
& \partial_{t} \Psi=\Delta_{\|} \Psi-\lambda \Psi-\partial_{\nu} \Phi, x \in \Gamma, \\
& \Psi(0)=l \\
& \left.\Phi\right|_{\Gamma}=\Psi
\end{aligned}
$$

with $\phi(t)=S_{\varepsilon}(t, 0, ; \omega) \phi_{0}$ and $m(h)=0$.

Proof. We write $(r(t), q(t))=\left(\phi_{1}(t)-\phi_{2}(t)-\Phi(t), \psi_{1}(t)-\psi_{2}(t)-\Psi(t)\right)$, where $\left(\phi_{i}(t), \psi_{i}(t)\right)(i=1,2)$ are two solutions to the problem (3.1) with $\left(\phi_{i}(0), \psi_{i}(0)\right)=$ $\left(\phi_{i}^{0}, \psi_{i}^{0}\right) \in \mathcal{A}_{\varepsilon}(\omega)$ and $(\Phi(t), \Psi(t))$ satisfies the linear system (5.4) with $\phi=\phi_{2}$ and $(h, l)=\left(\phi_{1}^{0}-\phi_{2}^{0}, \psi_{1}^{0}-\psi_{2}^{0}\right)$. Then $(r(t), q(t))$ solves

$$
\begin{aligned}
& \left(\varepsilon+A^{-1}\right) \partial_{t} r=\Delta r-<\partial_{\nu} r>_{\Gamma}-\overline{f\left(\phi_{1}\right)}+\overline{f\left(\phi_{2}\right)}+\overline{f^{\prime}\left(\phi_{2}\right) \Phi}, x \in G, \\
& r(0)=0, \\
& \partial_{t} q=\Delta_{\|} q-\lambda q-\partial_{\nu} r, x \in \Gamma, \\
& q(0)=0, \\
& \left.r\right|_{\Gamma}=q .
\end{aligned}
$$

It is easy to obtain $m(\Phi)=m(r)=0$ since $S_{\varepsilon}(t)$ maps $\mathbb{L}_{\varepsilon}^{\beta}$ into itself for $\forall \beta \in \mathbb{R}$. Taking the scalar product in $H$ of the first equation with $r$, we get

$$
\partial_{t}|(r(t), q(t))|_{\mathbb{L}_{\varepsilon}^{\beta}}^{2}+2|(r(t), q(t))|_{\mathbb{V}}^{2}+2 \lambda|q|_{\Gamma}^{2}=2\left(-\overline{f\left(\phi_{1}\right)}+\overline{f\left(\phi_{2}\right)}+\overline{f^{\prime}\left(\phi_{2}\right) \Phi}, r\right) .
$$


By the Hölder inequality and the Sobolev embedding theorem, for $s \in\left[\frac{6}{5}, 2\right]$ and its conjugate exponent $s^{*} \in[2,6]$, the right hand side of the above inequality may be estimated as

$$
\begin{aligned}
& 2\left(-\overline{f\left(\phi_{1}\right)}+\overline{f\left(\phi_{2}\right)}+\overline{f^{\prime}\left(\phi_{2}\right) \Phi}, r\right) \\
= & 2\left(-f\left(\phi_{1}\right)+f\left(\phi_{2}\right)+f^{\prime}\left(\phi_{2}\right)\left(\phi_{1}-\phi_{2}-r\right), r\right) \\
\leq & C|r|^{2}+2\left|f\left(\phi_{1}\right)-f\left(\phi_{2}\right)-f^{\prime}\left(\phi_{2}\right)\left(\phi_{1}-\phi_{2}\right)\right|_{L^{s}}|r|_{L^{s^{*}}} \\
\leq & |r|_{1}^{2}+C|r|_{-1}^{2}+C_{1}\left|f\left(\phi_{1}\right)-f\left(\phi_{2}\right)-f^{\prime}\left(\phi_{2}\right)\left(\phi_{1}-\phi_{2}\right)\right|_{L^{s}}^{2},
\end{aligned}
$$

where the fact $f^{\prime}\left(\phi_{2}\right) \geq-C$ is used. We continue to estimate the third term of (5.7). For $0<\delta<\frac{2}{s}-1$, we have

$$
\begin{aligned}
& \left|f\left(\phi_{1}\right)-f\left(\phi_{2}\right)-f^{\prime}\left(\phi_{2}\right)\left(\phi_{1}-\phi_{2}\right)\right|_{L^{s}}^{s} \\
\leq & C\left|\left(\left|\phi_{1}\right|^{2 p-3}+\left|\phi_{2}\right|^{2 p-3}+1\right)\left(\phi_{1}-\phi_{2}\right)^{2}\right|_{L^{s}}^{s} \\
\leq & C \int_{G}\left(\left|\phi_{1}\right|+\left|\phi_{2}\right|+1\right)^{s(2 p-2-\delta)}\left|\phi_{1}-\phi_{2}\right|^{s(1+\delta)} d x \\
\leq & C\left(\left|\phi_{1}\right|_{L^{s}}^{s(2 p-2-\delta)}+\left|\phi_{2}\right|_{L^{s}}^{s(2 p-2-\delta)}+1\right)\left|\phi_{1}-\phi_{2}\right|^{s(1+\delta)},
\end{aligned}
$$

where $s_{1}=\frac{2 s(2 p-2-\delta)}{2-s(1+\delta)}$. Therefore, Eq.(5.6) gives

$$
\begin{aligned}
& \partial_{t}|(r(t), q(t))|_{\mathbb{L}_{\varepsilon}^{\beta}}^{2} \\
\leq & C|r|_{-1}^{2}+C_{1}\left(\left|\phi_{1}\right|_{L^{s_{1}}}^{2(2 p-2-\delta)}+\left|\phi_{2}\right|_{\left.L^{s_{1}}-2-\delta\right)}^{2(2 p-2-\delta)}+1\right)\left|\phi_{1}-\phi_{2}\right|^{2(1+\delta)} \\
\leq & C|r|_{-1}^{2}+C_{1}\left(\left|\phi_{1}\right|_{L^{s_{1}}}^{2(2 p-2-\delta)}+\left|\phi_{2}\right|_{L^{s_{1}}}^{2(2 p-2)}+1\right)\left|\phi_{1}-\phi_{2}\right|_{1}^{1+\delta}\left|\phi_{1}-\phi_{2}\right|_{-1}^{1+\delta} .
\end{aligned}
$$

By (5.3) and (5.8), we have

$$
\begin{aligned}
|(r(1), q(1))|_{\mathbb{L}_{\varepsilon}^{\beta}}^{2} & \leq C \int_{0}^{1}\left(\left|\phi_{1}\right|_{L^{s_{1}}}^{2(2 p-2-\delta)}+\left|\phi_{2}\right|_{L^{s_{1}}}^{2(2 p-2-\delta)}+1\right)\left|\phi_{1}-\phi_{2}\right|_{1}^{1+\delta} d s|(h, l)|_{\mathbb{L}_{\varepsilon}^{\beta}}^{1+\delta} \\
& \leq C\left\{\int_{0}^{1}\left(\left|\phi_{1}\right|_{L^{s_{1}}}^{s_{2}}+\left|\phi_{2}\right|_{L^{s_{1}}}^{s_{2}}+1\right) d s|(h, l)|_{\mathbb{L}_{\varepsilon}^{\beta}}^{2(1+\delta)}\right. \\
& :=k^{2}|(h, l)|_{\mathbb{L}_{\varepsilon}^{\beta}}^{2(1+\delta)}
\end{aligned}
$$

where $s_{2}=4(2 p-2-\delta) /(1-\delta)$. Choosing $\bar{k}=\max \{k, 1\}$, which satisfies $E(\log \bar{k}(\omega))<$ $\infty$ by (4.11), we conclude the proof of Lemma 5.1]

We continue to verify that assumptions (2) and (3) of Theorem 2.10] hold in terms of the following lemma.

Lemma 5.2. For any $\left(\phi_{0}, \psi_{0}\right) \in \mathcal{A}(\omega)$, there exist a random variable $\bar{\alpha}_{1}(\omega)$ such that

$$
\alpha_{1}\left(D S\left(\omega ; \phi_{0}, \psi_{0}\right)\right) \leq \bar{\alpha}_{1}(\omega), \bar{\alpha}_{1}(\omega)>1, E\left(\log \bar{\alpha}_{1}(\omega)\right)<\infty,
$$

and another random variable $\bar{\omega}_{d}(\omega)$ such that

$$
\omega_{d}\left(D S\left(\omega ; \phi_{0}, \psi_{0}\right)\right) \leq \bar{\omega}_{d}(\omega), E \log \bar{\omega}_{d}(\omega)<0,
$$

where the notations $\omega_{d}$ and $\alpha_{1}$ are defined in Theorem 2.10. 
Proof. Multiplying the first equation of the variation system (5.1) scalarly in $H_{0}$ by $\Phi$, for $h \in H_{0}$, we have

$$
\partial_{t}|(\Phi, \Psi)|_{\mathbb{L}_{\varepsilon}^{\beta}}^{2}+|(\Phi, \Psi)|_{\mathbb{V}}^{2}+2 \lambda|\Psi|_{\Gamma}^{2} \leq 2 C|\Phi|_{-1}^{2}
$$

Hence, we get $|(\Phi(t), \Psi(t))|_{\mathbb{L}_{\varepsilon}^{\beta}} \leq|(h, l)|_{\mathbb{L}_{\varepsilon}^{\beta}} e^{C t}$. Since $\alpha_{1}\left(D S\left(\omega ; \phi_{0}, \psi_{0}\right)\right)$ is equal to the norm of $D S\left(\omega ; \phi_{0}, \psi_{0}\right) \in \mathcal{L}\left(\mathbb{L}_{\varepsilon}^{0}\right)$, we can choose a constant $\bar{\alpha}_{1}(\omega)=e^{C}$ such that

$$
\alpha_{1}\left(D S\left(\omega, \phi_{0}, \psi_{0}\right)\right) \leq \bar{\alpha}_{1}(\omega), E\left(\log \bar{\alpha}_{1}(\omega)\right)<\infty
$$

which gives (5.10). As for (5.11), we first write

$$
D S\left(\omega ; \phi_{0}, \psi_{0}\right)=\exp \left\{\int_{0}^{1} L(s, \phi(s), \psi(s)) d s\right\}
$$

and $L(s, \phi(s), \psi(s))(\Phi, \Psi)=\left(\left(\varepsilon+A^{-1}\right)^{-1}\left(\Delta \Phi-<\partial_{\nu} \Phi>_{\Gamma}-\overline{f^{\prime}(\phi) \Phi}\right), \Delta_{\|} \Psi-\lambda \Psi\right)$. Following Ref. 30, we have

$$
\omega_{d}\left(D S\left(\omega ; \phi_{0}, \psi_{0}\right)\right) \leq \sup _{\substack{\xi_{i} \in H \\ \xi_{i} \mid \leq 1, i=1, \cdots, d}} \exp \left\{\int_{0}^{1} \operatorname{tr}\left(L(s, \phi(s), \psi(s)) \circ Q_{d}(s)\right) d s\right\},
$$

where $Q_{d}(s)$ is the orthonormal projector in $H_{0} \times H(\Gamma)$ onto the space spanned by $\left(\Phi_{1}(s), \Psi_{1}(s)\right), \cdots,\left(\Phi_{d}(s), \Psi_{d}(s)\right)$, and $\left(\Phi_{i}(s), \Psi_{i}(s)\right)$ is the solution to the system (5.4) with $\left(\Phi_{i}(0), \Psi_{i}(0)=\left(\xi_{i}, \eta_{i}\right)\right.$.

Let $\left(\varphi_{i}(s), \psi_{i}(s)\right) \in \mathbb{V}, i \in \mathbb{N}$ be an orthonormal basis of $H_{0} \times H(\Gamma)$ such that $Q_{d}(s)\left(H_{0} \times H(\Gamma)\right)=\operatorname{Span}\left[\left(\varphi_{1}(s), \psi_{1}(s)\right), \cdots,\left(\varphi_{d}(s), \psi_{d}(s)\right)\right]$. Then

$$
\begin{aligned}
& \operatorname{tr}\left(L(s, \phi(s), \psi(s)) \circ Q_{d}(s)\right) \\
= & \sum_{i=1}^{d}\left(L(s, u(s))\left(\varphi_{i}(s), \psi_{i}(s),\left(\varphi_{i}(s), \psi_{i}(s)\right)\right)\right. \\
= & \sum_{i=1}^{d}\left(\Delta \varphi_{i}-<\partial_{\nu} \varphi_{i}>_{\Gamma}-\overline{f^{\prime}(\phi) \varphi_{i}},\left(\varepsilon+A^{-1}\right)^{-1} \varphi_{i}\right)+\sum_{i=1}^{d}\left(\Delta_{\|} \psi_{i}-\lambda \psi_{i}-\partial_{\nu} \varphi_{i}, \psi_{i}\right)_{\Gamma} \\
\leq & -C \sum_{i=1}^{d}\left|\varphi_{i}\right|_{2}^{2}+\sum_{i=1}^{d}\left(-\overline{f^{\prime}(\phi) \varphi_{i}},\left(\varepsilon+A^{-1}\right)^{-1} \varphi_{i}\right)-\sum_{i=1}^{d}\left|\psi_{i}\right|_{2, \Gamma}^{2}-\left(\partial_{\nu} \varphi_{i}, \psi_{i}\right)_{\Gamma} \\
\leq & -C \sum_{i=1}^{d}\left|\varphi_{i}\right|_{2}^{2}+\sum_{i=1}^{d}\left(-\overline{f^{\prime}(\phi) \varphi_{i}},\left(\varepsilon+A^{-1}\right)^{-1} \varphi_{i}\right)-\sum_{i=1}^{d}\left|\psi_{i}\right|_{2, \Gamma}^{2}+\sum_{i=1}^{d}\left|\psi_{i}\right|_{\frac{3}{2}, \Gamma}\left|\varphi_{i}\right| \\
\leq & -C \sum_{i=1}^{d}\left|\varphi_{i}\right|_{2}^{2}+\sum_{i=1}^{d}\left(-\overline{f^{\prime}(\phi) \varphi_{i}},\left(\varepsilon+A^{-1}\right)^{-1} \varphi_{i}\right)+C_{1} d^{2} .
\end{aligned}
$$

Writing $\zeta=\sum_{i=1}^{d}\left|\varphi_{i}\right|^{2}$ and choosing Hölder exponents $p_{n}=1+4 / n, q_{n}=2+$ $n / 2, n<4$, by the generalized Sobolev-Lieb-Thirring inequality (Ref. 30]

$$
|\zeta|_{L^{p_{n}}}^{p_{n}} \leq C \sum_{i=1}^{d}\left|\varphi_{i}\right|_{2}^{2}, \quad \sum_{i=1}^{d}\left|\varphi_{i}\right|_{2}^{2} \geq C_{1} d^{1+4 / n}-C_{2} d
$$


we have

$$
\begin{aligned}
& \operatorname{tr}\left(L(s, \phi(s), \psi(s)) \circ Q_{d}(s)\right) \\
& \leq-C \sum_{i=1}^{d}\left|\varphi_{i}\right|_{2}^{2}+\left(\left|f^{\prime}(\phi)\right| \zeta^{1 / 2},\left(\sum_{i=1}^{d}\left|\left(\varepsilon+A^{-1}\right)^{-1} \varphi_{i}\right|^{2}\right)^{1 / 2}\right)+C_{1} d^{2} \\
& \leq-C \sum_{i=1}^{d}\left|\varphi_{i}\right|_{2}^{2}+\left|f^{\prime}(\phi)\right|_{L^{q_{n}}}|\zeta|_{L^{p_{n}}}^{1 / 2}\left(\sum_{i=1}^{d}\left|\varphi_{i}\right|_{2}^{2}\right)^{1 / 2}+C_{1} d^{2} \\
& \leq-\frac{C}{2} \sum_{i=1}^{d}\left|\varphi_{i}\right|_{2}^{2}+C_{1}\left|f^{\prime}(\phi)\right|_{L^{q_{n}}}^{q_{n}}+C_{2} d^{2} \\
& \leq-C_{1} d^{1+4 / n}+C_{2} d++C_{3} d^{2}+C\left|f^{\prime}(\phi)\right|_{L^{q_{n}}}^{q_{n}} \\
& \leq-C_{1} d^{1+4 / n}+C_{2}+C\left|f^{\prime}(\phi)\right|_{L^{q_{n}}}^{q_{n}} .
\end{aligned}
$$

In the end, we deduce

$$
\omega_{d}\left(D S\left(\omega ; \phi_{0}, \psi_{0}\right)\right) \leq \exp \left\{-C_{1} d^{1+4 / n}+C_{2}+C \sup _{\left(\phi_{0}, \psi_{0}\right) \in \mathcal{A}_{\varepsilon}(\omega)} \int_{0}^{1}\left|f^{\prime}(\phi(t))\right|_{L^{q_{n}}}^{q_{n}} d t\right\}(5
$$

By the invariance of $\mathcal{A}_{\varepsilon}$ and (4.11), the third term in the exponent of the right hand side is finite. Denoting by $\bar{\omega}_{d}(\omega)$ the right hand side of (5.14) and choosing $d$ such that

$$
-C_{1} d^{1+4 / n}+C_{2}+C \sup _{\left(\phi_{0}, \psi_{0}\right) \in \mathcal{A}_{\varepsilon}(\omega)} \int_{0}^{1}\left|f^{\prime}(\phi(t))\right|_{L^{q_{n}}}^{q_{n}} d t<0,
$$

then we have $\omega_{d}(D S) \leq \bar{\omega}_{d}(\omega)$ and $E\left(\log \left(\bar{\omega}_{d}\right)\right)<0$.

In conclusion, we have the following result.

Theorem 5.3. If there exists $d$ such that (5.15) holds, then Hausdorff dimension of the random attractor for the Cahn-Hilliard system under stochastic dynamic boundary condition (1.5) is finite and the dimension is bounded by d, i.e., $d_{H}\left(\mathcal{A}_{\varepsilon}(\omega)\right)<d, P-$ a.s.

Remark 5.4. (Impact of stochastic dynamic boundary conditions.) A interesting problem is that if the dynamic boundary condition in Eq. (1.5) is replaced by

$$
\frac{1}{\varepsilon_{0}} d \psi=\left(\Delta_{\|} \psi-\lambda \psi-\partial_{\nu} \phi\right) d t+\sigma_{2} d W^{(2)}, x \in \Gamma,
$$

where $\varepsilon_{0}>0$ is a scaling parameter modeling the "intensity" of the time-derivative component in the dynamic boundary condition, then (5.15) has to be rewritten as

$$
-C_{1} d^{1+4 / n}+C_{2} \varepsilon_{0}^{\frac{4+n}{4-n}}+C_{3}+C \sup _{\left(\phi_{0}, \psi_{0}\right) \in \mathcal{A}_{\varepsilon}(\omega)} \int_{0}^{1}\left|f^{\prime}(\phi(t))\right|_{L^{q_{n}}}^{q_{n}} d t<0,
$$

which implies $d \sim \varepsilon_{0}^{\frac{n}{4-n}}$ and describes the impact of the dynamical boundary conditions on the global dynamics. 
We may think $\frac{1}{\varepsilon_{0}}$ as the intensity (relative importance) of the time derivative component in the dynamic boundary conditions (5.16). If $\varepsilon_{0}$ is very big (i.e., the time derivative term in the stochastic dynamic boundary conditions is very small), then the dimension is very big (Note that $n=1,2,3$ ). This says that the dimension of the random attractor increases as $\frac{1}{\varepsilon_{0}}$ decreases, namely, as the time derivative term becomes weaker.

However, in the limiting case $\varepsilon_{0}=\infty$, i.e., the stochastic dynamic boundary conditions reduce to the stochastic static boundary conditions,

$$
0=\left(\Delta_{\|} \psi-\lambda \psi-\partial_{\nu} \phi\right) d t+\sigma_{2} d W^{(2)}, x \in \Gamma,
$$

the dimension does not tend to infinity. Instead, we see that the stochastic static boundary conditions (5.17) do not have impact on the dimension. In fact, we can remove $\sum_{i=1}^{d}\left(\Delta_{\|} \psi_{i}-\lambda \psi_{i}-\partial_{\nu} \varphi_{i}, \psi_{i}\right)_{\Gamma}$ from the crucial equation (5.13), and thus, the stochastic static boundary conditions do not affect the later derivations and thus have no impact on the Hausdorff dimension of the random attractor.

\section{References}

[1] Arnold, L., "Random Dynamical System," Springer, Berlin, 1998.

[2] Blömker,D., Maier-Paape, S. and Wanner, T., "Spinodal decomposition for the Cahn-Hilliard-Cook equation," Commun. Math. Phys. 223, 553$582(2001)$.

[3] Blomker, D., Maier-Paape, S. and Wanner, T., "Second phase spinodal decomposition for the Cahn-Hilliard-Cook equation," preprint, 2006.

[4] Cahn, J. W. and Hilliard, J. E., "Free energy for a non-uniform system 1. Interfacial free energy," J. Chem. Phys. 2, 258-267(1958).

[5] Chueshov, I. and Schmalfuss, B. "Parabolic stochastic partial differential equations with dynamical boundary conditions." Differential Integral Equations 17 (2004), no. 7-8, 751-780.

[6] Crauel, H. and Flandoli, F., "Attractors for random dynamical systems," Prob. Th. and Related Firlds 100, 365-393(1994).

[7] Crauel, H., "Global random attractors are uniquely determined by attracting determinidtic compact set," Ann. Mat. Pura Appl. (4)176, 57-72(1999).

[8] Crauel, H., "Random point attractors versus random set attractors," J. London Math. Soc. (2)63, 413-427(2001). 
[9] Da Prato, G. and Debussche, A., "Stochastic Cahn-Hilliard equation," Nonlinear Analysis, Theory, Methods \& Application (2)26, 241-263(1996).

[10] Da Prato, G. and Zabczyk, J., "Stochastic Equations in Infinite Dimensions," In: Encyclopedia of Mathematics and its Apllication, 44, Cambridge: Cambridge University Press, 1992.

[11] Debussche, A., "Hausdorff dimension of a random invariant set," J. Math. Pures Appl. 77, 967-988 (1998).

[12] Desi, J. P., E. Sander and T. Wanner, "Complex transient patterns on the disk". Discrete and Continuous Dynamical Systems A 15 (2006), 1049-1078.

[13] Duan, J., H. Gao and B. Schmalfuss, "Stochastic Dynamics of a Coupled Atmosphere-Ocean Model". Stochastics and Dynamics 2 (2002), 357-380.

[14] Dudley, R. M., "Real Analysis and Probability", Cambridge Univ. Press, 2002.

[15] Elezovic, N. and A. Mikelic. "On the stochastic Cahn-Hilliard equation." Nonlinear Analysis 16 (1991), 1169-1200.

[16] Fischer, HP, Maass, Ph, and Dieterich, W. "Novel surface modes in spinodal decomposition". Phys. Rev. Lett. 79 (1997), 893-896.

[17] Fischer, HP, Maass, Ph, and Dieterich, W. "Diverging time and length scales of spinodal decomposition modes in thin flows". Europhys. Lett. 62 (1998), 49-54.

[18] Gameiro,M., Mischaikow, K. and Wanner, T. "Evolution of pattern complexity in the Cahn-Hilliard theory of phase separation". Acta Materialia 53(3), pp. 693-704, 2005. With .

[19] Huang, Z. and J. Yan, "Introduction to Infinite Dimensional Stochastic Analysis". Science Press/Kluwer Academic Pub., Beijing/New York, 1997.

[20] Kenzler, R., Eurich, F., Maass, Ph, Rinn, B., Schropp, J., Bohl, E. and Dieterich, W. "Phase separation in confined geometries: Solving the CahnHilliard equation with generic boundary conditions." Computer Physcs Communications 133 (2001), 139-157.

[21] Lin, G., Gao, H., Duan, J. and Ervin, V. "Asymptotic Dynamical Difference between the Nonlocal and Local Swift-Hohenberg Models," J. Math. Phys.41, 2077-2089(2000). 
[22] Maier-Paape, S. and Wanner, T. "Spinodal decomposition for the CahnHilliard equation in higher dimensions: Nonlinear dynamics". Archive for Rational Mechanics and Analysis 151(3), pp. 187-219, 2000.

[23] Miranville, A. and Zelik, S., "Exponential attractors for the Cahn-Hilliard equation with dynamic boundary conditions", Methematical methods in the applied sciences 28(6), 709-735(2005).

[24] Novick-Cohen, A., "The Cahn-Hilliard equation: mathematical and modeling perspectives", Adv. Math. Sci. Appl. 8(2), 965-985(1998).

[25] Oksendal, B., "Stochastic differential equation, An Introduction with Applications," 4th Edition, Springer-Verlag, Berlin, 1998.

[26] Racke, R. and Zheng, S., "The Cahn-Hilliard equation with dynamical boundary conditions", Advance in differential equations, 8, 83-110(2003).

[27] Rozovskii, B. L. Stochastic Evolution Equations. Kluwer Academic Publishers, Boston, 1990.

[28] Schmaluss, B., "Backward cocycles and attractors of stochastic differential equation", In "International Seminar on Applied Mathematics-Nonlinear Dynamics: Attractor Approximation and Global Behaviour," edited by N. Kokch, V. Reitmann, and T. Riedrich (Publisher,Technische Universität Dresden, 1992), 185-192.

[29] Schmaluss, B., "The stochastic attractor of the stochastic Lorenz system," Z. angew. Math. Phys. 48,951-975(1997).

[30] Temem, R. "Infinite-Dimensional Dynamical Systems in Mechanics and Physics," Springer-Verlag, New York, 1988.

[31] Waymire, E.and Duan, J. (Eds.), "Probability and Partial Differential Equations in Modern Applied Mathematics", IMA Volume 140, Springer-Verlag, New York, 2005.

[32] Wu, H. and Zheng, S., "Convergence to the equilibrium for the Cahn-Hilliard equation with dynamical boundary conditions", Journal of differential equations, 204(2), 511-531(2004). 